\title{
FTIR analysis of ash in wine
}

\author{
Tjaša $\operatorname{Jug}^{1}$, Saverio Boni ${ }^{2}$, and Tatjana Košmerl ${ }^{3}$ \\ ${ }^{1}$ Agricultural Chamber Nova Gorica, Pri hrastu 18, Nova Gorica, Slovenia \\ ${ }^{2}$ Steroglass srl, Strada Romano di Sopra, 2/C, 06132 - San Martino in Campo, Perugia (PG), Italy \\ ${ }^{3}$ Biotechnical faculty, University of Ljubljana, Jamnikarjeva 101, Ljubljana, Slovenia
}

\begin{abstract}
In a modern oenological laboratory determination of ash is a limiting factor regarding the speed of analysis, since functional FTIR analysis of ash are not common yet. Therefore 324 samples were analysed by FTIR spectroscopy on TDI Bacchus 3 as well as ISO 17025 accredited reference method (OIV-MAAS2-04: R2009) in two duplicates. FTIR spectra and reference data were uploaded into software for the calibration development and validation performed. Already the original TDI calibration was ready to use, showing satisfying results. However, we tried to improve it. On 324 spectra, we have looked for optimal wavelengths and mathematical models, which gave the best results. The developed calibration was then confirmed by method validation: repeatability, reproducibility and accuracy. The expanded measurement uncertainty calculated was $0.3 \mathrm{~g} / \mathrm{L}$, which I slightly higher than of the reference method used. We have found another advantage over classical method: there is no influence of the air pressure or moisture. Nevertheless, we were able to confirm that our reference method was free of systematic error induced by the influence of the weather. Anyway, we can conclude that analysis of ash in wine by FTIR spectroscopy is a promising, fast and reliable new method.
\end{abstract}

\section{Introduction}

Ash determination is an obligatory analysis for certified wines to be placed on the market. It is defined as all the inorganic matter remaining after igniting the residue left from the evaporation of must or wine [1]. Most laboratories, especially those running numerous sample tests, wish faster methods. Ash determination based on empirical equations relating ash content with the measurements of conductivity, seemed promising already more than 40 years ago [2], but they didn't get much practical use. The advantage of conductometric methods is in their precision $(1-2 \%)$, but the greatest problem concerning its application is the accuracy of results when it comes to such a complex medium with very different chemical composition as wine [2].

IR-spectroscopy is a modern analytical technique, based on measurement of IR spectra. As an indirect method, its results can only be as good as the results used in the calibration. Therefore, FTIR analyses need to overcome the weakness mentioned above anyway by developing a calibration model on few hundred samples.

Actually it is surprising that FTIR determination of ash is not a routine determination yet, since conductivity was one of the first reported parameters [3] with good correlation $\left(\mathrm{R}^{2}=0.95\right)$. Even in the latest review article [4] conductivity of wine is mentioned only once with the reference on the paper from 2004 [3]. We were able to find only one other paper dealing with the conductivity of wine, but even they report it only for spirit drink and not wine, and concluding FTIR data did not provide a good prediction for electrical conductivity [5].
One possible explanation is that the determination of inorganic compounds is more challenging compared to organic compounds owing to the lack of a direct relation between the element involved and the spectra obtained [6].

However, more likely to the authors' opinion the reason is not that the method was not promising, but more likely that there is no widely recognized conductivity's practical use. Even one of the larger inter-laboratory schemes in Europe (BIPEA) does not report its value.

So if ash in wine is theoretically correlated with conductivity and conductivity might be measured by FTIR spectroscopy, it seemed very attractive for us. We have received the basic calibration from the producer - TDI and tried to even improve it to the level of a practical use in a commercial lab.

\section{Materials and methods}

\subsection{Samples}

For the development of calibration and its verification samples analysed for the confirmation of the geographical origin for quality wine in Slovenia have been used (324 for development and 162 for verification). Also for method validation, except for its accuracy, samples of interlaboratory comparison BIPEA have been used.

\subsection{FTIR analysis}

FTIR analysis was performed on TDI Bacchus 3 FTIR instrument. FTIR spectra and reference data were uploaded into software for the calibration development and optimal bands have been searched. 
Table 1. Verification of different calibration with 162 samples: A: original calibration, no data, B: 2 wavelengths, first derivative, C: 4 wavelengths, first derivative, D: 4 wavelengths, centred average.

\begin{tabular}{|l|c|c|c|c|}
\hline Verification $\backslash$ calibration & A & B & C & D \\
\hline $\begin{array}{l}\text { Number of samples which not } \\
\text { achieving the criteria }\end{array}$ & 58 & 34 & 38 & 23 \\
\hline $\begin{array}{l}\text { Percent of samples which not } \\
\text { achieving the criteria (\%) }\end{array}$ & 36 & 20 & 24 & 14 \\
\hline $\begin{array}{l}\text { Minimal difference from classical } \\
\text { results }\end{array}$ & -1.0 & -0.5 & -0.5 & -0.6 \\
\hline $\begin{array}{l}\text { Maximal difference from classical } \\
\text { results }\end{array}$ & 0.7 & 39.6 & 22.8 & 1.1 \\
\hline
\end{tabular}

\subsection{Reference method}

As a reference, ash was determined in the samples by classical analysis performed according to OIV-MA-AS204: R2009 in two duplicates. The method is accredited by Slovenian accreditation (ISO 17025).

\subsection{Data analysis}

Multivariate analysis has been performed in QL Analyst software of TDI Company. Other calculations such as average, standard deviation, relative standard deviation (RSD) have been performed in LibreOffice 5.2.

\section{Results and discussion}

\subsection{Calibration development}

Data evaluation revealed a good correlation, even with the original calibration. Repeatability calculated according to OIV-OENO 10/2005 was $0.05 \mathrm{~g} / \mathrm{L}$, which is comparable to the repeatability of the OIV method $(0.06 \mathrm{~g} / \mathrm{L})$. Reproducibility of 46 control samples was 0.12 , which is very good - much lower than MAD. MAD was calculated as an average reproducibility limit of 22 inter-laboratory comparison (BIPEA) divided by $\sqrt{ } 2(\mathrm{MAD}=0.23)$. Accuracy was confirmed by comparing 12 ILC (BIPEA), where in 2 cases the results were questionable (z-score was 2.5 and 2.7 respectively), while in all other cases $z$ was lower than 2 (average $\mathrm{z}$ is 0.6 if we don't take into account questionable results).

Of course, we have tried to improve the accuracy of the method by additional selection of the wavelengths based of the spectra of 324 samples. Already with 2 wavelengths, we have obtained perfect results on 12 ILC samples all results report $\mathrm{z}$-value less than 2 . Even validation set looked optimistic: only 34 samples from 162 (20\%) reported difference between reference value and FTIR in absolute terms higher than $0.3 \mathrm{~g} / \mathrm{L}$ (Table 1 , method B).

Nevertheless, new problem has arisen: extreme false positive results, as can be observed from maximal difference. Of course, we have tried to add more wavelengths (Table 1, method $\mathrm{C}$ ), but the problem with false positive remained. The key improvement of the method was processing of the parameters: if we used first derivative (Table 1 , methods $\mathrm{B}$ and $\mathrm{C}$ ), several outliers were produced, but they are not if centred average is used in the calibration model (Table 1, method D).

The calibration based on centred average of 4 chosen wavelengths was chosen for validation.
Table 2. Repeatability of 6 different samples measured in 10 replicates.

\begin{tabular}{|l|c|c|c|c|c|c|}
\hline Replicate $\backslash$ sample & $\mathbf{1}$ & $\mathbf{2}$ & $\mathbf{3}$ & $\mathbf{4}$ & $\mathbf{5}$ & $\mathbf{6}$ \\
\hline 1 & 3.18 & 3.34 & 3.16 & 3.10 & 3.06 & 2.43 \\
\hline 2 & 3.24 & 3.33 & 3.14 & 3.07 & 3.09 & 2.38 \\
\hline 3 & 3.22 & 3.26 & 3.08 & 3.17 & 3.15 & 2.33 \\
\hline 4 & 3.24 & 3.25 & 3.16 & 3.17 & 3.08 & 2.35 \\
\hline 5 & 3.23 & 3.34 & 3.09 & 3.15 & 3.04 & 2.36 \\
\hline 6 & 3.19 & 3.34 & 2.99 & 3.09 & 3.08 & 2.38 \\
\hline 7 & 3.17 & 3.31 & 3.04 & 3.09 & 3.02 & 2.40 \\
\hline 8 & 3.22 & 3.41 & 3.02 & 3.02 & 3.08 & 2.41 \\
\hline 9 & 3.20 & 3.37 & 3.05 & 3.12 & 3.12 & 2.40 \\
\hline 10 & 3.26 & 3.38 & 3.09 & 3.05 & 3.03 & 2.44 \\
\hline Average & 3.22 & 3.33 & 3.08 & 3.10 & 3.08 & 2.39 \\
\hline Standard deviation & 0.03 & 0.05 & 0.06 & 0.05 & 0.04 & 0.03 \\
\hline RSD (\%) & 1 & 1 & 2 & 2 & 1 & 1 \\
\hline
\end{tabular}

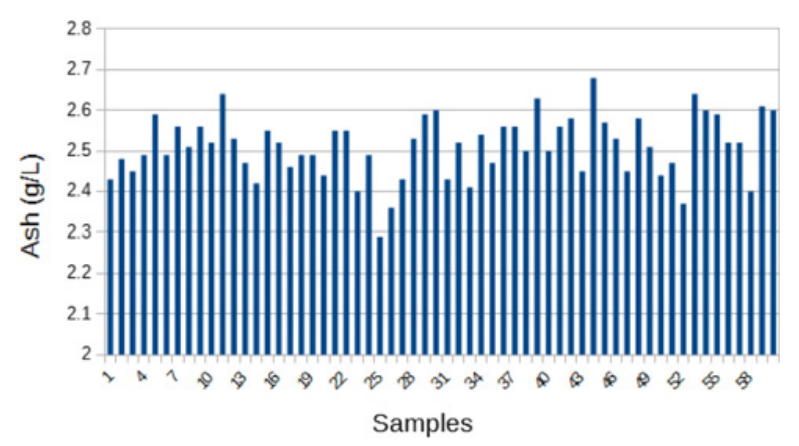

Figure 1. Reproducibility of the method over 2 month's period.

\subsection{Method validation}

The repeatability has been confirmed on 5 random samples, analysed in 10 replicates. The average of these 5 samples was reported as method repeatability.

Reproducibility has been calculated on one sample, analysed under the same conditions (60 measurements) in the period of 1.2. 17 until 29.3. 2017 and can be observed from Fig. 1. The calculated average value is 2.51 , standard deviation 0.08 and RSD 3\%.

The results of ILC analysis prove that the method is accurate: all ILC give z-score-values lower than 3, only one sample between 2 and 3 (questionable). From the data presented we have calculated measurement uncertainty of bias to be 0.14 .

The expanded measurement uncertainty calculated from reproducibility (accounts the same contributions as repeatability) and bias is $0.32 \mathrm{~g} / \mathrm{L}$, while expanded measurement uncertainty of reference method is $0.2 \mathrm{~g} / \mathrm{L}$.

Linearity doesn't need to be additionally confirmed, since calibration model is straight line with the correlation coefficient $\mathrm{R}^{2}=0.94$. Robustness of the method is confirmed through cross-validation performed by the software program itself, showing "performance/ deviation" ratio above 3. Moreover, less than 5\% samples were removed from the training set of 324 samples, proving the method selective and specific.

We are sure the method is "ready to use". However, for indirect methods such as FTIR, quality of the developed calibration depends mostly on the quality of the samples, used for the calibration. We believe that our data are of high quality - method accredited according to ISO 


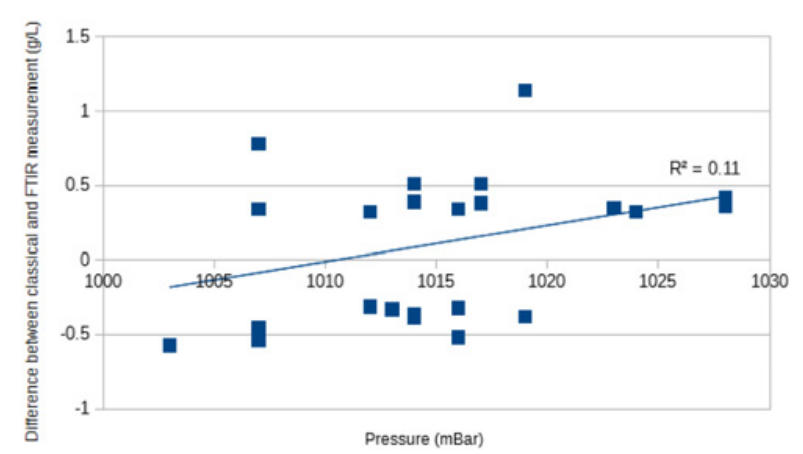

Figure 2. Influence of the air pressure on the difference between FTIR and classical measurement.

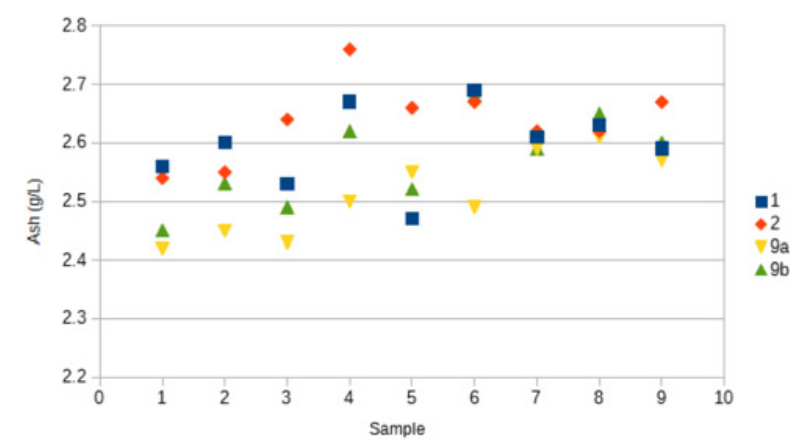

Figure 3. Influence of the order of weighing on the results.

17025, analysis done in duplicates, reproducibility and accuracy of the method are constantly monitored (accuracy at least once a month, reproducibility daily), results of ILC analysis of our reference method are very good (average $\mathrm{z}$ of 22 samples is 0.11 , no questionable results).

Some doubt has arisen that it might be susceptible to environmental conditions.

\subsection{Advantages of the FTIR method}

Apart from the obvious - speed, we have found another advantage over the classical method: no influence of environmental factors (moisture, air pressure). The influence might be observable because 100 multiply the weights to achieve results in $\mathrm{g} / \mathrm{L}$.

To evaluate the influence of the pressure on reference method we have compared the difference between classical and FTIR measurements, bigger than 0.3 in absolute terms, with average air pressure that day taken for meteorological station Bilje (Fig. 2).

We are not able to prove, which is actually very good information for our developed calibration: the samples used for the calibration do not contain systematic error due to air pressure.
There is another possible source of systematic error induced by the reference method: the samples might moisten during weighing. Again, it would not be a problem if we would not multiply the weights by 100 to achieve $\mathrm{g} / \mathrm{L}$. According to AOAC method of ash determination of vinegar, only two samples should be at the same time in the desiccator. There is no such demand in OIV method, so we are using one desiccator for up to 9 samples.

To test this we have analysed one sample in 9 replicates four different ways: 1: weighing samples one by one, so only one sample is in desiccator at the time, 2: 2 samples are in the desiccator at the same time and 9: all samples were in desiccator -2 sets, $a$ and $b$, where samples are randomly taken from desiccator. As seen from Fig. 3, weighing 9 samples at the same time has same influence, but it is similar to random error. Even if we cannot prove that FTIR method is better than classical one, because it is not susceptible to environmental factors, we can at least be sure, that we didn't introduce a systematic error that way either.

\section{Conclusions}

We can conclude that the determination of ash in wine by FTIR spectroscopy is a promising, fast and reliable new method. The best results are achieved if we use four wavelengths and a centred average. The validation of the method proves that it is already fit for purpose with expanded measurement uncertainty $0.3 \mathrm{~g} / \mathrm{L}$ already near classical method $0.2 \mathrm{~g} / \mathrm{L}$.

Apart from the obvious advantage of the method - the speed, we have found another advantage over the classical method: no influence of environmental factors such as air pressure and moisture. We were not able to prove that, however it confirms that calibration data systematic error free.

For the commercial use we still need few hundred samples to be sure, no outliers appear, but might be ready for accreditation next year.

\section{References}

[1] OIV - International Methods of Analysis of Wines and Musts (2017)

[2] T. Košmerl, D. Bavčar. Zb. Bioteh. Fak. Univ. Ljubl. 81, 325-334 (2003)

[3] C.-D. Patz, A. Blieke, R. Ristow, H. Dietrich. Anal. Chim. Acta 513, 81-89 (2004)

[4] C.A.T. dos Santos, R.N. Páscoa, J.A. Lopes. TrAC Trends Anal. Chem. 88, 100-118 (2017)

[5] B. Ozturk, D. Yucesoy, B. Ozen. Food Anal. Methods 5, 1435-1442 (2012)

[6] D. Cozzolino, W. Cynkar, N. Shah, P. Smith, Comput. Electron. Agric. 77, 81-85 (2011) 